\title{
Study on the Sign System of Underground Garage in Large Commercial Complex--Take Sign System of Underground Garage in Chengdu New Century Global Center as an Example
}

\author{
Qian Li, Fan Jiang, Ming Zhang, Yuxi Xie, Lingling Wei \\ College of Landscape Architecture, Sichuan Agricultural University, Wenjiang, Chengdu, Sichuan Province, \\ China
}

Keywords: large business districts; underground garage; sign system;

\begin{abstract}
At present, sign systems of large and medium-sized urban underground garages cannot guide users to find parking spaces conveniently. Under this situation, this paper selects the underground garage of Chengdu New Century Global Center as an example, and provides principles and directions for the design of underground garage sign system.
\end{abstract}

\section{Introduction}

With the rapid development of social economy, people are now in increasing demand for motor vehicles. In the face of increasing number of vehicles and limited ground spaces, underground garage provides a good way for people to park their cars. But when using underground parking space, due to the special underground environment, drivers are easy to suffer from psychological and physical fears and confusions which will directly affect the efficiency of parking and searching. Survey data shows that, the difficulty of parking in underground space is much higher than parking in ground space. As an important medium between space and human, underground garage sign system should be rationally designed to resolve difficulties in finding and remember parking lots and garage exits.

As the largest single building in Asia, Chengdu New Century Global Center is the largest commercial complex in Tianfu New Town with the construction area of about 1.76 million square meters. The full capacity of the underground garage is 5400 parking spaces, in addition to 5600 parking spaces on the ground (including 2800 temporary parking spaces). According to study of people's experience in underground garage, there are some problems in the sign system which has important guidance function. In view of these problems, the paper selects the sign system of underground garage in Chengdu New Century Global Center as an example, and explores how humanized and rational design can accurately guide drivers to quickly find parking space as well as entrances and exits, and put forward to the optimal design scheme.

\section{Current Situation of Chengdu Global Center Underground Garage}

Chengdu New Century Global Center is located in Dayuan group, Southern District of Chengdu. It is situated in Tianfu Avenue of Chengdu high-tech Zone and the junction of express highway circling the city. The center covers an area of about 1300 acres; the total construction area is about 1 million 760 thousand square meters; the plan view size of the main building is $500 * 400$ meters, while the height is about 100 meters. It is a multi-functional building which contains the central entertainment district, hotels, commercial area, office space and other places.

Parking space. Traffic flow of Global Center mainly comes from the north section of Tianfu Avenue, Yue Jin West Road and the north section of Yizhou Avenue. The traffic here is heavy. Chengdu New Century Global Center is a large commercial complex. It is composed of New Century Global Center, New Century Contemporary Art Center and Central Plaza. It has 15000 parking 
spaces in total; there are 5400 parking spaces in the underground garage of Chengdu New Century Global Center.

Traffic and visitors flows. Layout for Global Center underground garage is shown in Figure 1. Currently, the underground garage use colors to divide parking regions. Through the comparison of traffic flow in working days and holidays, we select holidays which have relatively heavy traffic flow, and make statistics in peak hours. During peak hours, as many as 138 vehicles can pass a single entrance every ten minutes, which brings about 309 people. Peak hour traffic flow is an important factor in sign system. Response speed and accuracy of drivers' understanding on signs will directly affect the normal traffic circulation in garage.

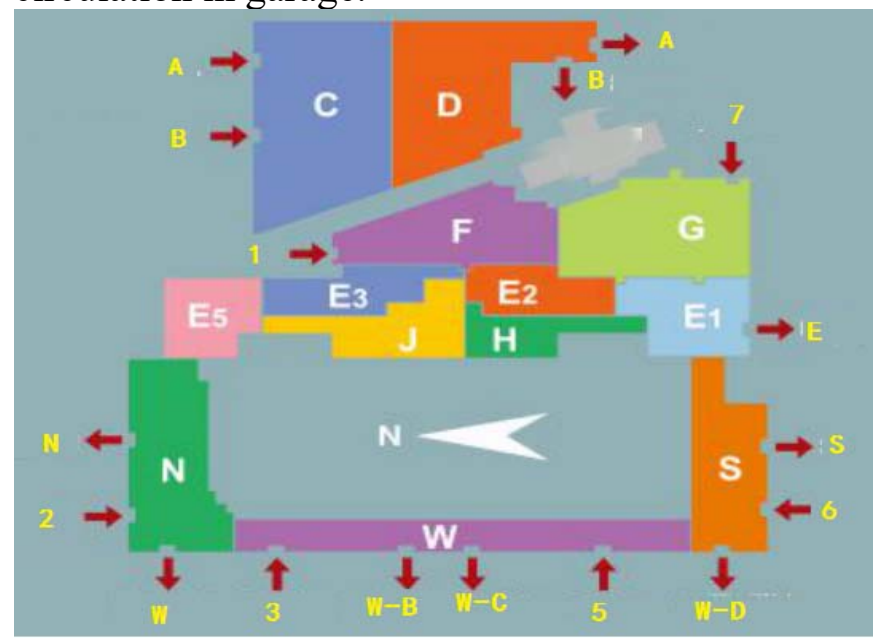

Figure 1 layout of underground garage of the global center
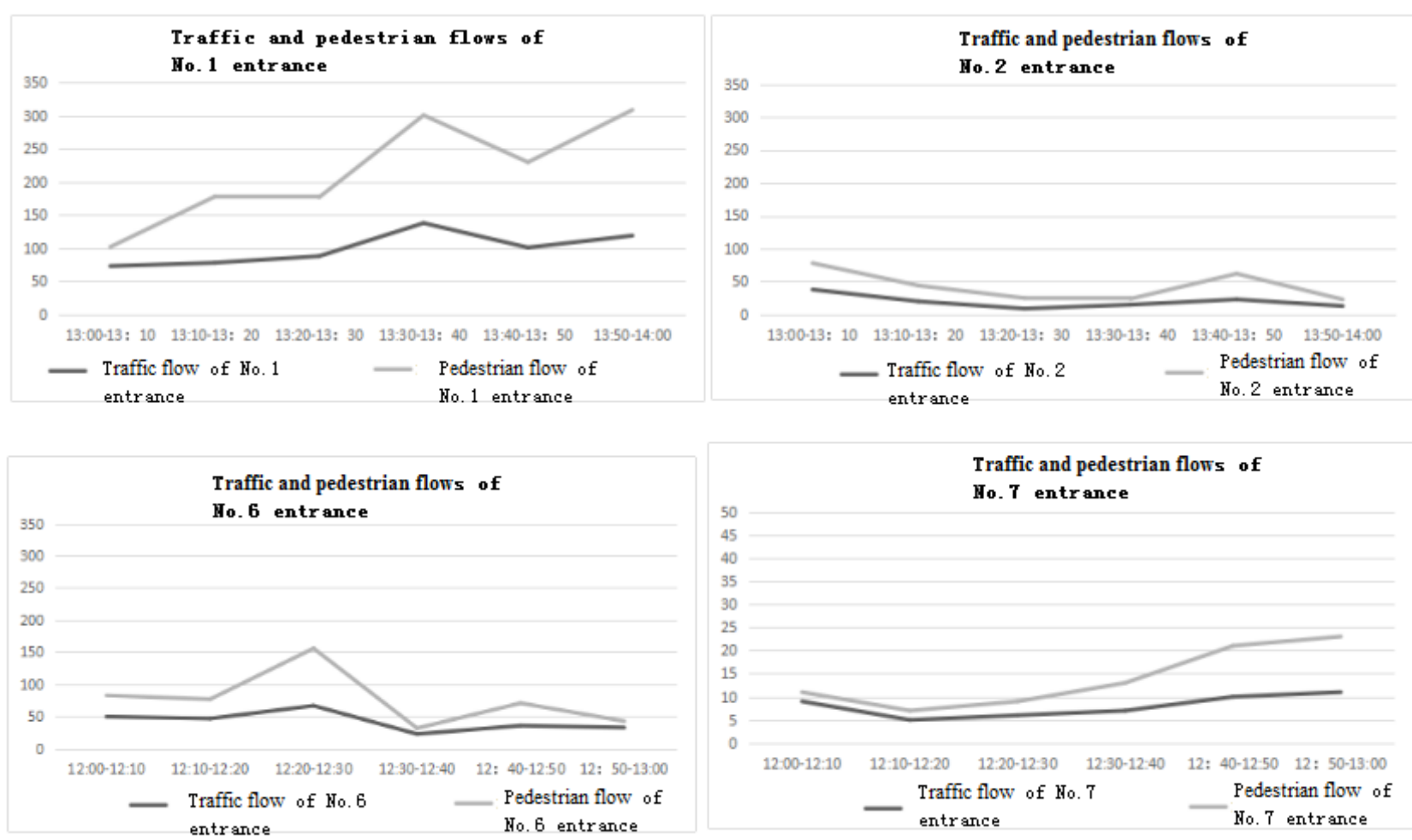

Figure 2 Traffic and pedestrian flows at different entrances and exits in a Saturday in summer

According to statistic data, in entrances of the commercial center, pedestrian flows fluctuate in line with traffic flow. Take statistical data on Saturday as an example. During 12:00 to 13:00, the highest value of pedestrian flow of No. 6 entrance reached about 155 at 12:20; during the same period, traffic flow of No. 7 entrance began to increase at 12:20, and reached a peak at 12:50. During 13:00-14:00, pedestrian flow in No. 1 entrance reached a peak at about 13: 50, the maximum value was about 310 
people. During the same period, the flow of pedestrian in No. 2 entrance reached a peak at about 13:00. As it can be seen from the data, the causes of such differences are as follows. No. 6 entrance is next to Chengdu freeway, resulting in large amount of external traffic flow; No. 1 entrance is located close to the front gate of global center; it is a relatively convenient entrance. From the perspective of human engineering, when people clearly know the location of destination, or they are in purposeful movement, they always choose the shortest path. [1]

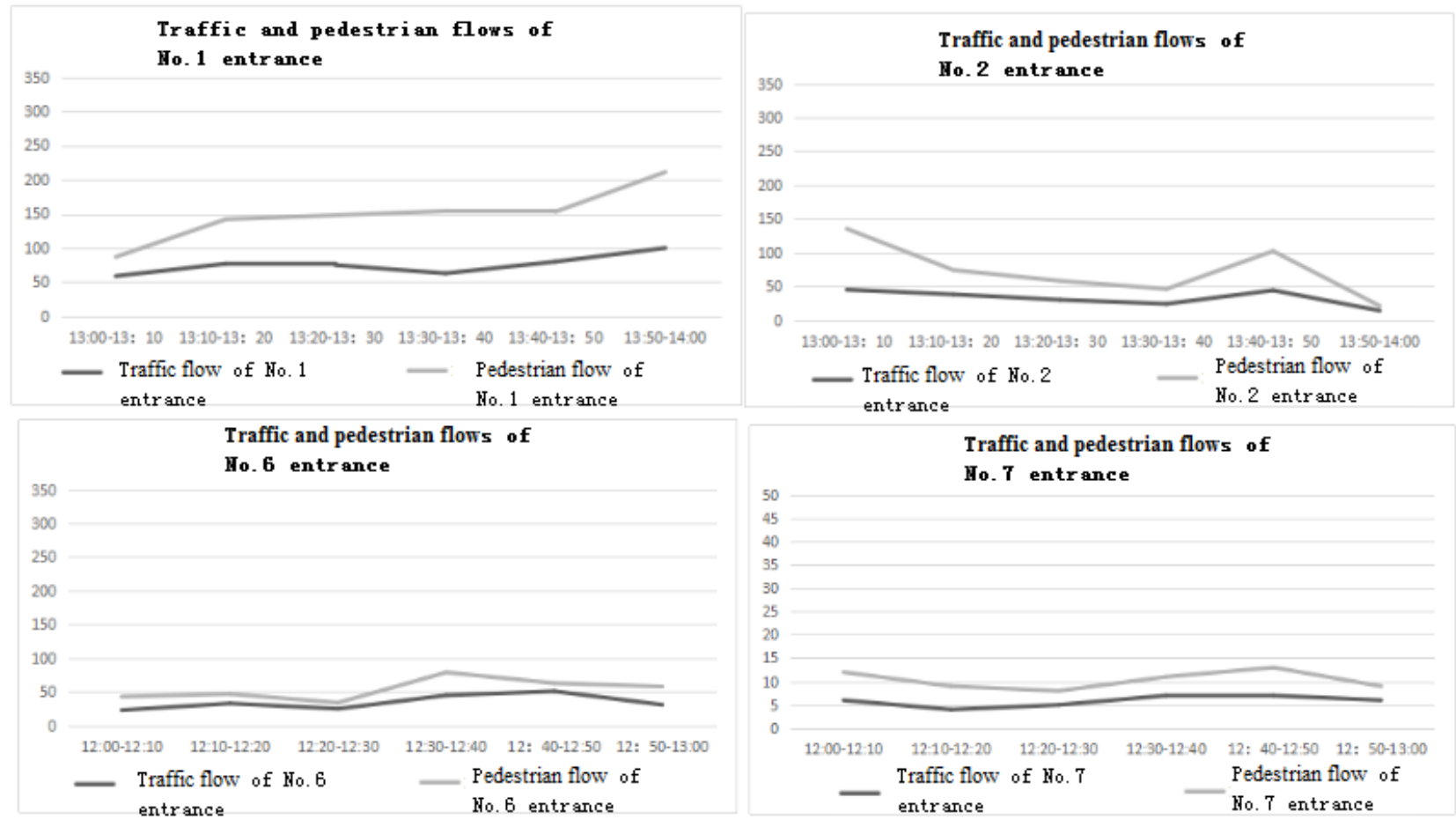

Figure 3 Traffic and pedestrian flow on a Sunday in winter

\section{Problems in the Sign System of Underground Garage of Chengdu Global Center}

Lack of systematic design. The design style of the sign system in underground garage should be consistent with the overall position of this region. Underground garage of the global center uses uniform colors; but there are different kinds of guidance symbols. Arrow symbols are in different sizes and shapes, which destroy the continuity of the sign system; sign boards also have different styles, fonts, graphics and layouts. Meanwhile, the garage sign system is not complete; logos of service facilities are missing. Therefore, the design concept of schematization is put forward combined with the usage of colors, in order to unify the theme of each regions and deepen the impressions of visitors.

Color in underground garage. The sign system of underground garage needs to accurately and quickly guide people to respond. Special experiments show that, when people enter a space, $75 \%$ of their initial impressions come from the feeling of color; identification of shapes goes afterwards. [2] In interior design, colors can bring people with more intense visual appeals compared with shapes, lines, materials, illumination conditions and so on. Simple and intuitive elements are more easily to be remembered; pure colors are easier to be distinguished and remembered than dull colors; bright, simple colors are easier to be distinguished and remembered than dark, complex colors.

The differences of color collocation can bring different visual psychological feelings. The overall color collocation will directly affect people's judgment and recognition of the space. The global center of the underground garage uses colors to divide parking areas, so as to promote the recognition effect. However, in the color collocation, there are problems which need to be tackled. For instance, in the garage, some similar colors are used at the same time, such as yellow and orange, blue and dark blue; such collocation can cause confusion in discrimination. Some choices of particular colors are not reasonable, either. The usage of deep green in large areas can bring people with depressed and 
gloomy psychological feelings; users may feel more insecurity in the underground space. On the other hand, too much colors used in the underground garage are extremely easy to cause confusion impressions. Moreover, the feeling is lost. A variety of light colors with high purity create the atmosphere of children's park, which does not match the modern and fashionable appearance of the global center as a commercial center.

In the plan sketch of the underground garage, the color of corresponding parking area is not consistent with the color actually painted in garage, which will mislead users. For instance, the color of $\mathrm{J}$ area in the sketch is different from color actually painted, and is similar to the color of $\mathrm{E}$ area.

Unreasonable size and location of guide signs. In the sign system of underground garage in global center, guide signs play the role of leading visitors. People may get lost due to the improper placement and irrational distribution of guide signs. The survey shows that, the lack of clear signs in intersections can lead to difficulties in choosing directions. According to above analyses on traffic and people flows, drivers tend to turn left under that situation, which brings about traffic congestion, insufficient parking spaces, and inadequate utilization of resources.

Underground garage of Chengdu global center is the underground garage of a comprehensive commercial building. Users are not familiar with the environment, and are lack of senses of security. In addition, parking spaces are not fixed; drivers need to look for parking spaces in the underground garage. Therefore, the demands for information integrity and information density are high. [4] The positions of signs should be rationalized set according to drivers' visual field.

As shown in Figure 2, the visual contact height which accounts for the largest proportion is 1.29 meters; the visual contact height of $85 \%$ vehicles are 1.18 meters; the average visual contact height is about 1.24 meters. 25\% visual contact heights are less than 1.20 meters, the current highway engineering technical standard in China. [5]

According to the type of cars, corresponding standards are as follows.

Table 1 Minimum turning radius of vehicles

\begin{tabular}{ll}
\hline type & Minus radius of turning circle $(\mathrm{m})$ \\
\hline mini car & 4.5 \\
Small car & 6 \\
light vehicle & $6.5-8.0$ \\
\hline
\end{tabular}

It is shown that, $h^{\prime}$ is the area where the mark should be placed, $s^{\prime}$ means the minimum distance between the vehicle and the wall, that is, the minimum radius of turning circle for vehicles.

Based on the field investigation and experimental experience, when a driver is searching for parking spaces, and the speed is about $20 \mathrm{~km} / \mathrm{h}$ to $30 \mathrm{~km} / \mathrm{h}$, the best angle for drivers in vertical direction is 15 degrees, so that the scope of h' should be from 2.30 meters to 3.56 meters.

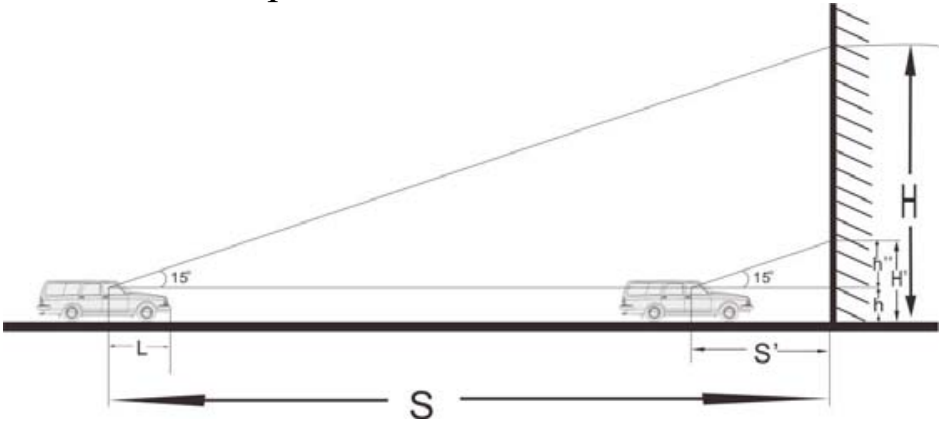

Figure 4 Visual contact heights of different distances

Miscellaneous information. The underground garage should provide enough information to guide drivers, but complicated and difficult information may hinder drivers from making right judgment. In the sign system of global center underground garage, the sign patterns are simple and clear enough, while the image is not difficult to understand. When an indication information contains 
too much words, the cognitive time of drivers will increase. In addition to the influences of driving speed, it will be difficult for drivers to make correct and effective judgment.

Incomplete sign system. A complete set of garage sign system should contain signs for garage and service facilities, layout plan for the whole building and layout plan for the garage, integrated service hall, corridor signage, signs for public service facilities, as well as entrances and exits for the shopping mall and the garage. After investigation, it is found that the current underground garage sign system of global center does not contain signs for public service facilities. Subjects in this survey claim that it is difficult for them to find specific parking spaces; most drivers expressed that they had no impression on functional spaces.

Chengdu New Century Global Center is a shopping center. Customers can go from the shopping mall to the underground parking lot from outside and inside paths. But in these paths which must be passed, there are no clear signs on underground garage information. It is difficult for users to rapidly understand the content of these signs, and a large number of instructions are only provided in the elevator. They rarely appear in the commercial space. Since the global center is a large building, and there is no clear direction on sign system, people are difficult to find the entrance of the underground garage if there are no indications from working staffs.

Other problems. Besides above deficiencies, there are following problems in the management operation of underground garage. (1) The toll centers of underground garage are difficult to find. There are no signs on toll centers. Although there are self-help charging machines, customers are hard to find them without signs and tips. (2) The environment is dark and dim; almost no public service facilities can be found in the garage. (3) No working staff in duty room. These problems can be solved to a certain extent by optimizing the sign system.

\section{Principles in Sign System Design}

Rational. Under the most basic requirements of underground garage sign system, designers need to make out more humanized and rational plans on positions of hanging signs, layout and font of characters, and garage indicating information in commercial spaces according to the model of human behaviors.

Systematic. Designers should consider the commercial style of the global center, and make the underground parking space become a continuation of the commercial space. Underground garage and the global center should be a whole unity. The systematism of sign system reflects in the complete sign system. Except for signs in underground garage, continuous guidance information should also be provided in the mall to help users to find the underground garage.

Emotional. A large number of studies have shown that, emotional memories are more distinct, vivid, profound and situational than general memory. Thus, in the optimal design of sign system, designers can strengthen emotional elements, use distinct themes and matched theme colors in different regions to emphasize emotion information conveyed by each theme. Through the combination of themes and colors, the underground garage can provide people with different feelings and experiences. When finding their parking spaces, people can have fun and feel relaxed.

\section{Acknowledgement}

Fund Project: This paper is the outcome of the research, Design of Visual Sign System in Underground Parking Space, which is supported by Foundation for the Projects of Sichuan Landscape Architecture Center. (Project No. 2015YB05).

\section{References}

[1] S.H. Liu, Ergonomics and Interior Design, 2nd Edition. China Architecture \& Building Press, 2004. 
[2] B.H. Wang, L.L. Lin, Study on the design of color and environment of underground garage--take the underground garage of Puerta America hotel in Madrid, Spain as an example, J. Modern Decoration (Theory). 2(2013)

[3] L. Wang, Urban complex - a new commercial real estate model, J. Urban Development. 3 (2008) $83-84$

[4] L.J. Yu, X.D. Pan, Design of guidance system for motor vehicle in underground garage of urban complex, J. Journal of Transportation Engineering and Information. 12 (2012).

[5] T.R. Jing, Y.F. He, J.Z. Wang, J.S. Zhang, The determination of minimum radius of drivers' sight line and the vertical curve of the road, J. East China Highway. 6 (1984).

[6] R.H. Wang, New concept on packaging design based on brand strategy, J. Packaging Engineering. $12(2009) 223$. 